\title{
Electron Tunneling Through Fluid Solvents
}

\author{
Nicholas Prokopuk,* Kyung-ah Son, ${ }^{+}$and Chad Waltz \\ Chemistry and Materials Division, Naval Air Warfare Center, China Lake, California \\ 93555 and ${ }^{+}$The Jet Propulsion Laboratory, Pasadena, California 91109
}

\section{Supporting Information}

S1. List of relative barrier heights in $\mathrm{eV}$ for the various solvents

Formamide $\quad 0.74$

Water 0.93

Ethylene glycol $\quad 1.22$

Ethanol $\quad 1.54$

Methanol 1.56

1-Propanol 1.68

Benzyl alcohol 2.06

1-Butanol 2.63

Pyridine $\quad 1.83$

1,2-Dichloroethane $\quad 2.07$

Dimethyl sulfoxide $\quad 2.12$

2-Methyltetrahydrofuran $\quad 2.13$

1,1,2,2-Tetrachloroethane $\quad 2.15$

Nitromethane $\quad 2.21$

Acetonitrile $\quad 2.50$

$N, N$-Dimethylformamide $\quad 2.51$

Ethylacetate $\quad 3.03$

Bromobenzene $\quad 3.05$

n-Decane $\quad 3.34$

Toluene $\quad 3.38$

n-Hexane $\quad 3.46$

cyclohexane $\quad 3.56$

Benzene $\quad 3.85$

1,4-Dioxane $\quad 3.87$

Carbontetrachloride $\quad 4.16$ 